\title{
Crosstalk-Free Widesense Nonblocking Multicast Photonic Switching Networks
}

\author{
Hung Q. Ngo, Thanh-Nhan Nguyen, Duc T. Ha \\ Computer Science and Engineering, \\ 201 Bell Hall, \\ State University of New York at Buffalo, \\ Amherst, NY 14260, USA. \\ \{hungngo,nguyen9,ducha\}@cse.buffalo.edu
}

\begin{abstract}
We analyze crosstalk-free widesense nonblocking multicast multi-log networks. Widesense nonblocking strikes a balance between strictly nonblocking and rearrangeably nonblocking both in terms of cost-effectiveness and route establishment efficiency. The problem is important as cost-effective and efficient crosstalk-free multicast photonic switching networks are likely to play an important role at the optical Internet core in the future.

Particularly, for the first time in the literature we derive conditions for the $d$-ary multi-log network to be crosstalk-free multicast widesense nonblocking under the window algorithm for any given window size. Several interesting by-products follow from our approach and result. Firstly, our result allows for computing the best window size minimizing the fabric cost, showing that the multi-log network is a very good candidate for crosstalk-free multicast switching architectures. Secondly, our analytical approach - partly based on applying the celebrated König-Egevarý theorem - also gives a much simpler proof of the known case when the network is not required to be crosstalk-free. Thirdly, for window sizes of 1 and $d^{n}$, we re-discovered known results for the multi-log multicast networks under the so-called fanout constraints.
\end{abstract}

Index Terms-Multicast, Photonic Switching Fabrics, Crosstalk-Free, Widesense Nonblocking

\section{INTRODUCTION}

To meet the ever-increasing bandwidth demands, an alloptical Internet backbone is inevitable [1], and has been demonstrated to be a very real possibility in the near future [2], [3]. Many applications require multicast as the fundamental communication primitive. Thus, cost-effective and route establishment-efficient multicast-capable photonic switching fabrics are crucial in realizing all-optical networks. Researchers in recent years have spent a lot of effort on analyzing and designing photonic switching architectures at all levels of granularities: space, wavelength, down to the optical-burst/packet level [4]-[11]. Whichever the switchinggranularity is, space-switching architectures remain the core component. (For example, at the wavelength-level, expensive wavelength converters are placed at appropriate places in a space-domain switch.) Consequently, designing cost-effective multicast-capable photonic switching networks (or optical cross-connects) in the space domain is an important research problem.

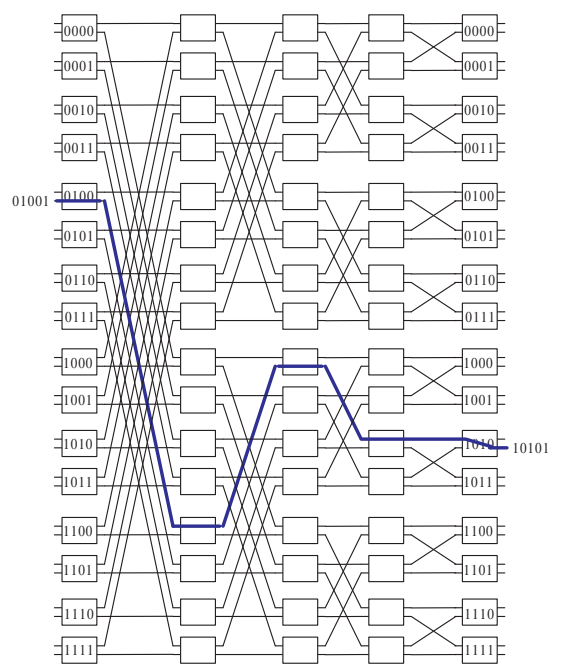

Fig. 1. The inverse Banyan network $\mathrm{BY}^{-1}(5)$

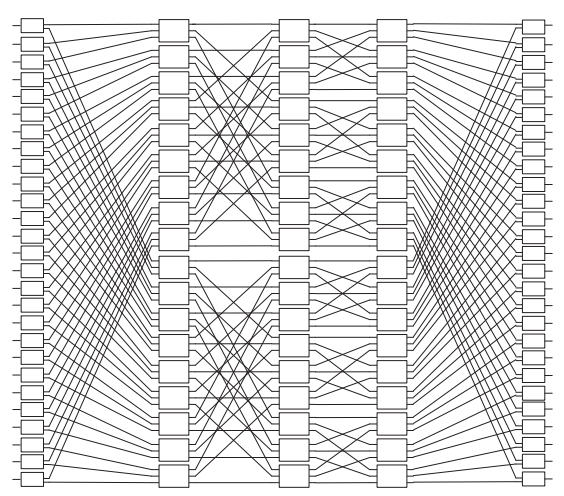

Fig. 2. The $\log _{3}(27,0,2)$ network

One of the major choices for designing multicast photonic switching networks is the multi-log networks, because they have small depth $(O(\log N))$, absolute signal loss uniformity, and good fault tolerance [6], [12]-[17]. Hereafter, we use $\log _{d}(N, 0, m)$ to denote a $d$-ary multi-log network with $m$ vertically stacked inverse Banyan planes $\left(\mathrm{BY}^{-1}(n)\right)$, as illustrated in Figures 1 and 2. 
The switching elements (SE) of multi-log networks can naturally be constructed with perhaps the most widely studied and commercially available optical components: the directional couplers [18]-[20] (for the $d=2$ case). However, directional couplers and many other optical switching elements suffer from optical crosstalk between interfering channels, which is one of the major obstacles in designing cost-effective switches [21]-[23]. Although crosstalk is a complicated phenomenon to model, it has been shown that reducing the number of SEs shared between active routes in a switch will reduce crosstalk. In particular, if no two active routes share any common SE, then the design is said to be crosstalk-free [13], [16].

There are three nonblockingness-degrees of a switch: rearrangeably nonblocking (RNB), widesense nonblocking (WSNB), and strictly nonblocking (SNB). The reader is referred to [24] for their precise definitions. SNB switches require minimal effort in routing a new request at the price of higher switch cost (in terms of the number of SEs). RNB switches have the lowest cost but require rearranging existing connections, complicating route establishment algorithms, and disrupting existing flows. WSNB switches strike a balance between SNB and RNB switches both in terms of costeffectiveness and routing-establishment efficiency.

Putting together all of the above issues, this paper addresses the problem of analyzing crosstalk-free WSNB multicast multi-log networks. In particular, for the first time in the literature we derive conditions for the $\log _{d}(N, 0, m)$ network to be crosstalk-free multicast widesense nonblocking under the window algorithm [25], [26] for any window size. Several interesting by-products follow from our approach and result. Firstly, our result allows for estimating the best window size minimizing the fabric cost. Secondly, our approach - based on a simple application of the celebrated König-Egevarý theorem - also gives a much simpler proof of the case when the network is not required to be crosstalk free. Thirdly, for window sizes of 1 and $d^{n}$, we re-discovered known results for SNB multi-log multicast networks under the so-called fanout constraints [10].

Prior to this paper, various necessary and/or sufficient conditions have been derived for the $\log _{d}(N, 0, m)$ networks to be $f$-cast SNB [10], multicast WSNB [27], [28], $f$-cast RNB [29], [30], unicast SNB under various crosstalk constraints [10], [13]. However, there was no result on multicast WSNB crosstalk-free multi-log networks.

The rest of the paper is organized as follows. Section II presents basic notations, the window algorithm, and a simple algebraic view of $\log _{d}(N, 0, m)$ networks, which are used throughout the paper. Section III derives conditions for $\log _{d}(N, 0, m)$ to be crosstalk-free multicast WSNB, along with several consequences including computing the optimal window size. Section IV concludes the paper with a few remarks regarding our proof technique and its application in deriving conditions where crosstalk-free is not required.

\section{Preliminaries}

Throughout this paper, for any positive integers $k, d$, let $[k]$ denote the set $\{1, \ldots, k\}, \mathbb{Z}_{d}$ denote the set $\{0, \ldots, d-1\}$ which can be thought of as $d$-ary "symbols", $\mathbb{Z}_{d}^{k}$ denote the set of all $d$-ary strings of length $k$. Let $N=d^{n}$. We consider the $\log _{d}(N, 0, m)$ network, which denotes the stacking of $m$ copies of the $d$-ary inverse Banyan network $\mathrm{BY}^{-1}(n)$ with $N$ inputs and $N$ outputs. Label the inputs and outputs of $\mathrm{BY}^{-1}(n)$ with $d$-ary strings of length $n$. Specifically, each input $\mathbf{x} \in \mathbb{Z}_{d}^{n}$ and output $\mathbf{y} \in \mathbb{Z}_{d}^{n}$ have the form $\mathbf{x}=x_{1} \cdots x_{n}$, $\mathbf{y}=y_{1} \cdots y_{n}$, where $x_{i}, y_{i} \in \mathbb{Z}_{d}, \forall i \in[n]$.

Also, label the $d \times d$ SEs in each of the $n$ stages of $\mathrm{BY}^{-1}(n)$ with $d$-ary strings of length $n-1$. An input $\mathbf{x}$ (resp. output $\mathbf{y})$ is connected to the SE labeled $\mathbf{x}_{1 . . n-1}$ in the first stage (resp. $\mathbf{y}_{1 . . n-1}$ in the last stage). In general, the unique path $R(\mathbf{x}, \mathbf{y})$ in $\mathrm{BY}^{-1}(n)$ from an arbitrary input $\mathbf{x}$ to an arbitrary output $\mathbf{y}$ is exactly the following: input $\mathbf{x}=x_{1} x_{2} \ldots x_{n-1} x_{n}$ to stage-1 SE $x_{1} x_{2} \ldots x_{n-1}$ to stage-2 SE $y_{1} x_{2} \ldots x_{n-1}$ to stage-3 SE $y_{1} y_{2} \ldots x_{n-1}, \ldots$, to stage- $n$ SE $y_{1} y_{2} \ldots y_{n-1}$, to output $\mathbf{y} y_{1} y_{2} \ldots y_{n-1} y_{n}$.

Now, consider two unicast requests $(\mathbf{a}, \mathbf{b})$ and $(\mathbf{x}, \mathbf{y})$. From the observation above, routes $R(\mathbf{a}, \mathbf{b})$ and $R(\mathbf{x}, \mathbf{y})$ share a common SE iff there is some $j \in[n]$ such that $b_{1 . . j-1}=$ $y_{1 . . j-1}$ and $a_{j . . n-1}=x_{j . . n-1}$. In this case, the two routes intersect at a stage- $j$ SE. (Note: two requests' routes may intersect at more than one SE.)

For any two $d$-ary strings $\mathbf{u}, \mathbf{v} \in \mathbb{Z}_{d}^{l}$, let $\operatorname{PRE}(\mathbf{u}, \mathbf{v})$ denote the longest common prefix, and $\operatorname{SUF}(\mathbf{u}, \mathbf{v})$ denote the longest common suffix of $\mathbf{u}$ and $\mathbf{v}$, respectively. For example, if $\mathbf{u}=0100110$ and $\mathbf{v}=0101010$, then $\operatorname{PRE}(\mathbf{u}, \mathbf{v})=010$ and $\operatorname{SUF}(\mathbf{u}, \mathbf{v})=10$. The following proposition immediately follows.

Proposition II.1. Let $(\mathbf{a}, \mathbf{b})$ and $(\mathbf{x}, \mathbf{y})$ be two unicast requests. Their routes $R(\mathbf{a}, \mathbf{b})$ and $R(\mathbf{x}, \mathbf{y})$ in (the same copy of) $B Y^{-1}(n)$ share at least a common $S E$ iff

$$
\left|\operatorname{SUF}\left(\mathbf{a}_{1 . . n-1}, \mathbf{x}_{1 . . n-1}\right)\right|+\left|\operatorname{PRE}\left(\mathbf{b}_{1 . . n-1}, \mathbf{y}_{1 . . n-1}\right)\right| \geq n-1 .
$$

The Window Algorithm: the so-called window algorithm for routing multicast requests in a multi-log network was proposed in [26] and extended in [25]. In its present form, the window algorithm with window size $d^{t}$ can be desribed as follows. Given any integer $t, 0 \leq t \leq n$, divide the outputs into windows of size $d^{t}$ each. Each window consists of outputs sharing a prefix of length $n-t$, for a total of $d^{n-t}$ windows. Given a new multicast request $(\mathbf{a}, B)$, where $\mathbf{a}$ is an input and $B$ is a subset of outputs, let $B_{w}$ be the set of outputs in $B$ lying in the $w$ th window. Route the sub-request $\left(\mathbf{a}, B_{i}\right)$ separately on the same $\mathrm{BY}^{-1}(n)$ plane.

\section{Main Results}

The following theorem is the main result of the paper. Its consequences and the proof technique's implications are explored later in the section and in Section IV. 
Theorem III.1. The d-ary multi-log network $\log _{d}(N, 0, m)$ is crosstalk-free widesense nonblocking under the window algorithm with window size $d^{t}$ if

$m \geq\left\{\begin{array}{l}d^{n-2 t}+t d^{n-t}(d-1), \text { when } t<n / 2, \\ d^{n-t}[(d-1)(n-t)-1]+d^{t}+1, \text { when } t=n / 2, \\ d^{n-t}[(d-1)(n-t)-1]+d^{t}-d^{2 t-n-2}(d-1)+1, \\ \quad \text { when } t>n / 2 .\end{array}\right.$

Proof: Let $(\mathbf{a}, B)$ be an arbitrary multicast request to be routed using the window algorithm with window size $d^{t}$. Since each part of a multicast request belonging to the same window can be routed independently from each other, we can assume that $B=\left\{\mathbf{b}^{(1)}, \ldots, \mathbf{b}^{(k)}\right\}$ where all the $\mathbf{b}^{(p)}(p \in[k])$ belong to the same window. Specifically, the $\mathbf{b}^{(p)}$ share a common prefix of length $n-t$.

For each $i \in\{0, \ldots, n-1\}$, let $A_{i}$ be the set of inputs $\mathbf{x}$ other than $\mathbf{a}$, where $\mathbf{x}_{1 . . n-1}$ shares a suffix of length exactly $i$ with $\mathbf{a}_{1 . . n-1}$. Formally, define

$$
A_{i}:=\left\{\mathbf{x} \in \mathbb{Z}_{d}^{n}-\{\mathbf{a}\} \mid \operatorname{SUF}\left(\mathbf{x}_{1 . . n-1}, \mathbf{a}_{1 . . n-1}\right)=i\right\} .
$$

For each $j \in\{0, \ldots, n-1\}$, let $B_{j}$ be the set of outputs other than those in B which share a prefix of length exactly $j$ with some member of $B$, namely

$B_{j}:=\left\{\mathbf{y} \in \mathbb{Z}_{d}^{n}-B \mid \exists p \in[k], \operatorname{PRE}\left(\mathbf{y}_{1 . . n-1}, \mathbf{b}_{1 . . n-1}^{(p)}\right)=j\right\}$.

Note that $\left|A_{i}\right|=d^{n-i}-d^{n-1-i}$, for all $0 \leq i \leq n-1$, and $\left|B_{j}\right|=d^{n-j}-d^{n-1-j}$ for all $0 \leq j \leq n-t-1$. Moreover, for each $j \leq n-t-1, B_{j}$ is the disjoint union of precisely $d^{n-j-t}-d^{n-1-j-t}$ windows of size $d^{t}$ each. When $j \geq n-t$, $B_{j}$ belongs to the same window which $B$ belongs.

Suppose the network $\log _{d}(N, 0, m)$ already had some routes established. Consider a $\mathrm{BY}^{-1}(n)$ plane which blocks the new request $(\mathbf{a}, B)$. There must be one route $R(\mathbf{x}, \mathbf{y})$ on this plane for which $R(\mathbf{x}, \mathbf{y})$ and $R\left(\mathbf{a}, \mathbf{b}^{(p)}\right)$ share an SE, for some $p \in$ $[k]$. Note that $R(\mathbf{x}, \mathbf{y})$ could be part of a multicast request from input $\mathbf{x}$, but we only need the branch $(\mathbf{x}, \mathbf{y})$ of this multicast request to block $(\mathbf{a}, B)$. Assemble one blocking branch $(\mathbf{x}, \mathbf{y})$ per blocking plane into a set $S$. Then, the number of blocking planes is $|S|$. This way, if $(\mathbf{x}, \mathbf{y})$ and $\left(\mathbf{x}, \mathbf{y}^{\prime}\right)$ are both in $S$ then $\mathbf{y}$ and $\mathbf{y}^{\prime}$ must belong to different windows.

The strategy is to find and upperbound $U$ for $|S|$, where $U$ is independent of $(\mathbf{a}, B)$; then, $U+1$ will be the sufficient number of planes so that the network is nonblocking.

By proposition II.1, each $(\mathbf{x}, \mathbf{y}) \in S$ must belong to some $A_{i} \times B_{j}$ where $i+j \geq n-1$. Partition $S$ into two parts:

$$
\begin{aligned}
& S_{1}=\left\{(\mathbf{x}, \mathbf{y}) \in S \mid \mathbf{y} \in B_{j}, j \leq n-t-1\right\} \\
& S_{2}=\left\{(\mathbf{x}, \mathbf{y}) \in S \mid \mathbf{y} \in B_{j}, j \geq n-t\right\}
\end{aligned}
$$

To upperbound $|S|=\left|S_{1}\right|+\left|S_{2}\right|$, we bound $\left|S_{1}\right|$ and $\left|S_{2}\right|$ separately.

We first derive an upperbound for $\left|S_{1}\right|$. As noted earlier, for each $j \leq n-t-1$ there are precisely $d^{n-j-t}-d^{n-1-j-t}$ windows in $B_{j}$, each of which contains $d^{t}$ outputs. Each output $\mathbf{y}$ in a window of $B_{j}$ can only be part of at most one $(\mathbf{x}, \mathbf{y}) \in$ $S_{1}$. Furthermore, each input $\mathbf{x} \in A_{i}$ with $i \geq n-j-1$ can only be part of at most one $(\mathbf{x}, \mathbf{y})$ with $\mathbf{y}$ in a given window of $B_{j}$. Consequently,

$$
\begin{aligned}
& \left|\left\{(\mathbf{x}, \mathbf{y}) \in S_{1}: \mathbf{y} \in B_{j}\right\}\right| \\
\leq & \left(d^{n-j-t}-d^{n-1-j-t}\right) \min \left\{d^{t}, \sum_{i \geq n-j-1}\left|A_{i}\right|\right\} \\
= & \left(d^{n-j-t}-d^{n-1-j-t}\right) \min \left\{d^{t}, d^{j+1}-1\right\}
\end{aligned}
$$

Summing the above over $j=0, \ldots, n-t-1$ we obtain

$$
\begin{aligned}
\left|S_{1}\right| & =\sum_{j=0}^{n-t-1}\left|\left\{(\mathbf{x}, \mathbf{y}) \in S_{1}: \mathbf{y} \in B_{j}\right\}\right| \\
& \leq \sum_{j=0}^{n-t-1}\left(d^{n-j-t}-d^{n-1-j-t}\right) \min \left\{d^{t}, d^{j+1}-1\right\} \\
& = \begin{cases}d^{n-2 t}-d^{t}+t d^{n-t}(d-1) & t<n / 2 \\
d^{n-t}(d-1)(n-t)-d^{n-t}+1 & t \geq n / 2\end{cases}
\end{aligned}
$$

Next, we derive an upperbound for $\left|S_{2}\right|$. By definition, for each $(\mathbf{x}, \mathbf{y}) \in S_{2}$ we have $\mathbf{y} \in B_{j}$ where $j \geq n-t$. As noted before, $B_{j}$ belongs to the same window as that of $B$ for all such $j$. Construct a bipartite graph $G=(L \cup R, E)$ where $L=\bigcup_{i=0}^{n-1} X_{i}$ is the set of inputs, $R=\bigcup_{j=n-t}^{n-1} B_{j}$, and $E$ is the union of all $A_{i} \times B_{j}$ for which $j \geq n-t$ and $i+j \geq n-1$. Then, $S_{2}$ forms a matching of $G$. The classic König-Egevarý theorem [31] says that the size of a maximum matching in a bipartite graph $G$ is equal to the size $\nu(G)$ of a minimum vertex cover of $G$. Thus,

$$
\left|S_{2}\right| \leq \nu(G),
$$

So, to upperbound $\left|S_{2}\right|$ we will find an upperbound for the minimum vertex cover of $G$. Note that $G$ and thus $\nu(G)$ depends on $(\mathbf{a}, B)$. We will need to find a bound independent of $(\mathbf{a}, B)$, which will be the worst-case bound.

Since the $B_{j}(j \geq n-t)$ belong to the same window, we have $|R|=d^{t}-k$. We can assume that $k<d^{t}$, otherwise the graph $G$ is empty and we reach the trivial case $\left|S_{2}\right|=0$. Let $r=\left\lfloor\log _{d} k\right\rfloor$, then $d^{r} \leq k<d^{r+1}$ and $0 \leq r \leq t-1$. Consider the following vertex sets of $G$, indexed by integers $q$ from $n-t$ to $n$.

$$
\begin{aligned}
C_{n-t} & =\bigcup_{j=n-t}^{n} B_{j} \\
C_{q} & =\left(\bigcup_{i=n-q}^{n-1} A_{i}\right) \cup\left(\bigcup_{j=q}^{n-1} B_{j}\right), \text { for } n-t<q \leq n
\end{aligned}
$$

Firstly, we claim that $C_{q}$ is a vertex cover of $G$ for any $q \in$ $\{n-t, \ldots, n\}$. When $q=n-t, C_{q}$ is a vertex cover since $C_{q}=R$ and every edge is incident to some vertex in $R$. When $n-t<q \leq n-1$, if there is an edge $(\mathbf{x}, \mathbf{y})$ of $G$ which is not covered by $C_{q}$ then $(\mathbf{x}, \mathbf{y}) \in A_{i} \times B_{j}$ where $i \leq n-q-1$ and $j \leq q-1$. However, in that case $i+j \leq(n-q-1)+(q-1)=$ 
$n-2$, contradicting the definition of $G$. Lastly, when $q=n$ $C_{q}=\bigcup_{i=0}^{n-1} A_{i}=L$ and thus $C_{q}$ is certainly a vertex cover.

We already know $\left|C_{n-t}\right|=d^{t}-k$. For $n-t<q \leq n$, note that $\left|\bigcup_{j=q}^{n-1} B_{j}\right|$ is the number of outputs other than those in $B$ sharing a prefix of length at least $q$ with one of the $\mathbf{b}^{(p)}, p \in[k]$. For each $p \in[k]$, the number of outputs sharing a prefix of length at least $q$ with $\mathbf{b}^{(p)}$ (other than $\mathbf{b}^{(p)}$ itself) is at most $d^{n-q}-1$. Hence, by the union bound

$$
\left|\bigcup_{j=q}^{n-1} B_{j}\right| \leq k\left(d^{n-q}-1\right) .
$$

Thus, for $n-t<q \leq n$, we have

$$
\begin{aligned}
\left|C_{q}\right| & =\left|\bigcup_{i=n-q}^{n-1} A_{i}\right|+\left|\bigcup_{j=q}^{n-1} B_{j}\right|=d^{q}-1+\left|\bigcup_{j=q}^{n-1} B_{j}\right| \\
& \leq d^{q}-1+k\left(d^{n-q}-1\right) .
\end{aligned}
$$

To this end, consider three cases as follows. The main idea is to choose the vertex cover $C_{q_{0}}$ with minimum size depending on the relationship between $t$ and $n$.

Case 1: $2 t \leq n$. In this case, set $q_{0}=n-t$. Then,

$$
\nu(G) \leq\left|C_{n-t}\right|=d^{t}-k \leq d^{t}-1 .
$$

Case 2: $2 t \geq n+1$ and $k \geq d^{2 t-n-1}-d^{2 t-n-2}+1$. In this case, set $q_{0}=n-t$. Then,

$$
\nu(G) \leq\left|C_{n-t}\right|=d^{t}-k \leq d^{t}-d^{2 t-n-1}+d^{2 t-n-2}-1 .
$$

Case $32 t \geq n+1$ and $k \leq d^{2 t-n-1}-d^{2 t-n-2}$. This means $r \leq 2 t-n-2$.

Case 3a If $r=2 t-n-2$, then setting $q_{0}=t-1$ we get

$$
\begin{aligned}
\nu(G) & \leq\left|C_{t-1}\right| \leq d^{t-1}-1+k\left(d^{n-t+1}-1\right) \\
& \leq d^{t-1}-1+\left(d^{2 t-n-1}-d^{2 t-n-2}\right)\left(d^{n-t+1}-1\right) \\
& =d^{t}-d^{2 t-n-1}+d^{2 t-n-2}-1 .
\end{aligned}
$$

Case 3b If $r \leq 2 t-n-3$, then, setting $q_{0}=\left\lfloor\frac{n+r+1}{2}\right\rfloor$, we obtain

$$
\begin{aligned}
\nu(G) & \leq\left|C_{q_{0}}\right| \leq d^{\left\lfloor\frac{n+r+1}{2}\right\rfloor}-1+k\left(d^{n-\left\lfloor\frac{n+r+1}{2}\right\rfloor}-1\right) \\
& \leq d^{\left\lfloor\frac{n+r+1}{2}\right\rfloor}-1+\left(d^{r+1}-1\right)\left(d^{n-\left\lfloor\frac{n+r+1}{2}\right\rfloor}-1\right) \\
& =d^{\left\lceil\frac{n+r+1}{2}\right\rfloor}+d^{\left\lfloor\frac{n+r+1}{2}\right\rfloor}-d^{r+1}-d^{n-\left\lfloor\frac{n+r+1}{2}\right\rfloor} .
\end{aligned}
$$

The function

$$
g(r):=d^{\left\lceil\frac{n+r+1}{2}\right\rceil}+d^{\left\lfloor\frac{n+r+1}{2}\right\rfloor}-d^{r+1}-d^{n-\left\lfloor\frac{n+r+1}{2}\right\rfloor}
$$

is non-decreasing in $r$ when $0 \leq r \leq 2 t-n-3$, hence

$$
\nu(G) \leq g(2 t-n-3)=2 d^{t-1}-d^{2 t-n-2}-d^{n-t+1} .
$$

The upperbound of $\nu(G)$ in case $3 \mathrm{~b}$ is smaller than those in cases 2 and $3 \mathrm{a}$ (which are equal). Hence, when $2 t>n$

$$
\nu(G) \leq d^{t}-d^{2 t-n-1}+d^{2 t-n-2}-1 .
$$

Putting inequalities (1), (2), (3), and (4) together, we get

$$
|S| \leq d^{n-2 t}+t d^{n-t}(d-1)-1 \text { when } t<n / 2,
$$

$$
|S| \leq d^{n-t}[(d-1)(n-t)-1]+d^{t} \text { when } t=n / 2,
$$

and $|S| \leq d^{n-t}[(d-1)(n-t)-1]+d^{t}-d^{2 t-n-2}(d-1)$ when $t>n / 2$. The theorem follows because one more $\mathrm{BY}^{-1}(n)$ plane is needed to route the new request $(\mathbf{a}, B)$.

We next illustrate the strength of Theorem III.1 by deriving as a consequence several known results.

In [10], necessary and sufficient conditions for $\log _{d}(N, 0, m)$ to be SNB under fanout constraints were proved. If the input stage (the demux stage) does not have fanout capability, then every multicast request must be routed entirely through the same Banyan plane. This corresponds to the window size $d^{n}$. It is crucial to notice that our analysis does not at all depend on how the requests were routed, as long as branches belonging to the same window are routed through the same plane. Hence, when the window size is $d^{n}$ we actually have a SNB condition under the constraint that the input stage does not have fanout capability. The following corollary matches the necessary and sufficient condition derived in [10]. We only need to "plug-in" the value $t=n$ in Theorem III.1.

Corollary III.2. If the input (demux) stage does not have fanout capability, then $\log _{d}(N, 0, m)$ is strictly nonblocking if $m \geq d^{n}-d^{n-1}+d^{n-2}$.

Next, when both stages have fanout capability, each unicast branch of a multicast request can be routed independently from each other. This corresponds exactly to the case when the window size is 1 , i.e. $t=0$. Similarly, our result gives a matching condition as the necessary and sufficient condition in [10].

Corollary III.3. When both the demux stage and the Banyan stage have fanout capability, the $\log _{d}(N, 0, m)$ network is strictly non-blocking if $m \geq d^{n}$.

The previous two corollaries illustrate a counter-intuitive point: when the network has less capability, it might also cost less in the SNB sense. This is because being SNB requires the network to be able to find a new route for any existing network state, no matter how peculiar. The more capability the network has, the easier it is to construct a network state where more planes are blocking planes!

Last but not least, Theorem III.1 allows for computing the best window size minimizing the number of switching elements in the network. A little calculus reveals that asymptotically picking $t \approx\left(n+\log _{d} n\right) / 2$ is best. In this case, the number of Banyan planes is $\Theta\left(n d^{n / 2}\right)=\Theta(\sqrt{N \log N})$. Each Banyan planes has depth $O(\log N)$ and crosspoint complexity $O(N \log N)$. In our optical switching sense, crosspoint complexity corresponds roughly to the number of directional couplers.

Corollary III.4. Using multi-log networks, it is possible to construct $N \times N$ multicast WSNB crosstalk-free switching networks with depth $O(\log N)$ crosspoint complexity $\Theta\left((N \log N)^{3 / 2}\right)$. 
This complexity is within a sub-linear factor of the optimal, known in the non-crosstalk-free case to be $\Theta(N \log N)$ ) but existing optimal constructions require larger depths [32].

\section{Concluding Remarks}

When there is no crosstalk-free constraint, only linkblocking takes effect. We have the following analogue of Proposition II.1.

Proposition IV.1. Let $(\mathbf{a}, \mathbf{b})$ and $(\mathbf{x}, \mathbf{y})$ be two unicast requests. Then their corresponding routes $R(\mathbf{a}, \mathbf{b})$ and $R(\mathbf{x}, \mathbf{y})$ in (the same copy of) $B Y^{-1}(n)$ share at least a common link if and only if

$$
\left|\operatorname{SUF}\left(\mathbf{a}_{1 . . n-1}, \mathbf{x}_{1 . . n-1}\right)\right|+\left|\operatorname{PRE}\left(\mathbf{b}_{1 . . n-1}, \mathbf{y}_{1 . . n-1}\right)\right| \geq n .
$$

Following the proof of Theorem III.1, define $S$ similarly and note that each $(\mathbf{x}, \mathbf{y}) \in S$ must belong to some $A_{i} \times B_{j}$ where $i+j \geq n$ (note the lowerbound of $n$ instead of $n-1$ as in Theorem III.1). Completely analogous reasoning leads to another proof of the following known result, which was derived recently in [28] with a much longer argument.

Theorem IV.2. The d-ary multi-log network $\log _{d}(N, 0, m)$ is widesense nonblocking under the window algorithm with window size $d^{t}$ if the number $m$ of Banyan planes is at least

$$
\left\{\begin{array}{l}
d^{n-1-2 t}+t d^{n-t-1}(d-1), \text { when } t<\frac{n-1}{2}, \\
d^{n-t-1}(d-1)(n-t-1)-d^{n-t-1}+d^{t}+1, \text { when } t=\frac{n}{2}, \\
d^{n-t-1}[(d-1)(n-t-1)-1]+d^{t}-d^{2 t-n-1}(d-1)+1, \\
\quad \text { when } t>n / 2 .
\end{array}\right.
$$

\section{REFERENCES}

[1] B. Mukherjee, "WDM optical communication networks: progress and challenges," IEEE J. Select. Areas Commun., vol. 18, no. 10, pp. 1810$1824,2000$.

[2] D. Bishop, C. Giles, and G. Austin, "The Lucent LambdaRouter: MEMS technology of the future here today," IEEE Communication Magazine, pp. 75-79, Mar 2002.

[3] Lucent Technologies Press Release, "Lucent Technologies engineer and scientists set new fiber optic transmission record," 2002. http://www.lucent.com/press/0302/020322.bla.html.

[4] Y. Yang and J. Wang, "Designing WDM optical interconnects with full connectivity by using limited wavelength conversion," IEEE Trans. Comput., vol. 53, no. 12, pp. 1547-1556, 2004.

[5] Y. Yang and J. Wang, "WDM optical switching networks using sparse crossbars," in Proceedings of the 23th Annual Joint Conference of the IEEE Computer and Communications Societies (INFOCOM'2004, Hong Kong), IEEE, 2004.

[6] X. Jiang, H. Shen, M. M. ur Rashid Khandker, and S. Horiguchi, "Blocking behaviors of crosstalk-free optical banyan networks on vertical stacking," IEEE/ACM Transactions on Networking, vol. 11, no. 6, pp. 982-993, 2003.

[7] H. Q. Ngo, D. Pan, and Y. Yang, "Optical switching networks with minimum number of limited range wavelength converters," in Proceedings of the 24rd Annual Joint Conference of the IEEE Computer and Communications Societies (INFOCOM), vol. 2, (Miami, Florida, U.S.A.), pp. 1128-1138, IEEE, March 2005.

[8] H. Q. Ngo, D. Pan, and C. Qiao, "Nonblocking WDM switches based on arrayed waveguide grating and limited wavelength conversion," in Proceedings of the 23rd Conference of the IEEE Communications Society (INFOCOM), (Hong Kong, China), IEEE, 2004.
[9] H. Q. Ngo, "WDM switching networks, rearrangeable and nonblocking $[w, f]$-connectors," SIAM Journal on Computing, vol. 35, no. 3, pp. 766785, 2005-2006.

[10] Y. Wang, H. Q. Ngo, and X. Jiang, "Strictly nonblocking $f$-cast $d$-ary multilog networks under fanout and crosstalk constraints," in Proceedings of the 2008 International Conference on Communications (ICC), (Bejing, China), IEEE, 2008.

[11] C. Qiao and M. Yoo, "Optical burst switching (OBS) - a new paradigm for an optical internet," Journal of High Speed Networks, vol. 8, no. 1, pp. 69-84, 1999.

[12] C.-T. Lea, "Muti- $\log _{2} n$ networks and their applications in high speed electronic and photonic switching systems," IEEE Transactions on Communications, vol. 38, no. 10, pp. 1740-1749, 1990.

[13] M. M. Vaez and C.-T. Lea, "Strictly nonblocking directional-couplerbased switching networks under crosstalk constraint," IEEE Trans. Comm., vol. 48, pp. 316-323, Feb 2000.

[14] F. Hwang, "Choosing the best $\log _{2}(n, m, p)$ strictly nonblocking networks," IEEE Transactions on Communications, vol. 46, pp. 454-455, Dec 1998.

[15] D.-J. Shyy and C.-T. Lea, " $\log _{2}(n, m, p)$ strictly nonblocking networks," IEEE Transactions on Communications, vol. 39, no. 10, pp. 1502-1510, 1991.

[16] G. Maier and A. Pattavina, "Design of photonic rearrangeable networks with zero first-order switching-element-crosstalk," IEEE Trans. Comm., vol. 49, pp. 1248-1279, Jul 2001.

[17] C.-T. Lea and D.-J. Shyy, "Tradeoff of horizontal decomposition versus vertical stacking in rearrangeable nonblocking networks," IEEE Transactions on Communications, vol. 39, pp. 899-904, 1991.

[18] T. E. Stern and K. Bala, Multiwavelength Optical Networks: A Layered Approach. Upper Saddle River, NJ: Prentice Hall PTR, 1999.

[19] J.-C. Wu and T.-L. Tsai, "Low complexity design of a wavelengthselective switch using raman amplifiers and directional couplers," in GLOBECOM, 2006.

[20] B. Mukherjee, Optical Communication Networks. New York, NY: McGraw-Hill, 1997.

[21] M. Vaez and C.-T. Lea, "Wide-sense nonblocking Banyan-type switching systems based on directional couplers," IEEE J. Select. Areas Commun., vol. 16, pp. 1327-1332, Sep 1998.

[22] V. Chinni, T. Huang, P.-K. Wai, C. Menyuk, and G. Simonis, "Crosstalk in a lossy directional coupler switch," J. Lightwave Technol., vol. 13, no. 7, pp. 1530-1535, 1995.

[23] D.Li, "Elimination of crosstalk in directional coupler switches," Optical Quantum Electron.,, vol. 25, no. 4, pp. 255-260, 1993.

[24] F. K. Hwang, The mathematical theory of nonblocking switching networks. River Edge, NJ: World Scientific Publishing Co. Inc., 2004.

[25] W. Kabacinski and G. Danilewicz, "Wide-sense and strict-sense nonblocking operation of multicast multi- $\log _{2} n$ switching networks," IEEE Transactions on Communications, vol. 50, pp. 1025-1036, Jun 2002.

[26] Y. Tscha and K.-H. Lee, "Yet another result on multi- $\log _{2} n$ networks," IEEE Transactions on Communications, vol. 47, pp. 1425-1431, Sep 1999.

[27] F.K.Hwang and B.-C. Lin, "Wide-sense nonblocking multicast $\log _{2}(n, m, p)$ networks," IEEE Transactions on Communications, vol. 51, pp. 1730-1735, Oct 2003.

[28] G. Danilewicz, "Wide-sense nonblocking $\log _{\mathrm{d}}(\mathrm{n}, 0, \mathrm{p})$ multicast switching networks," IEEE Transactions on Communications, vol. 55, no. 11, pp. 2193-2200, 2007.

[29] X. Jiang, A. Pattavina, and S.Horiguchi, "Rearrangeable $f$-cast multi$\log 2 n$ networks," IEEE Transactions on Communications, 2007. to appear.

[30] H. Q. Ngo, Y. Wang, A. Le, and X. Jiang, "Better necessary conditions for rearrangeably nonblocking f-cast d-ary multi-log networks under fanout and crosstalk constraints," in Proceedings of the 2008 International Workshop on High Performance and Highly Survivable Routers and Networks (HPSRN), (Sendai, Japan), IEEE, Mar 2008.

[31] L. Lovász and M. D. Plummer, Matching theory. Amsterdam: NorthHolland Publishing Co., 1986. Annals of Discrete Mathematics, 29.

[32] H. Q. Ngo and D.-Z. Du, "Notes on the complexity of switching networks," in Advances in Switching Networks (D.-Z. Du and H. Q. Ngo, eds.), vol. 5 of Network Theory and Applications, pp. 307-367, Kluwer Academic Publishers, 2001. 